\title{
Radiological Considerations for Top-Up Operation of the Storage Ring
}

\author{
Harold J. Moe \\ February 26, 1998
}

\section{General}

Radiological considerations for the operation of the storage ring prior to top-up operation have been discussed in the document (MOE 94). This document was prepared to serve as the technical basis for the hazard analysis considerations and the statements in the APS Safety Assessment Document (SAD) dealing with shielding adequacy and other radiological considerations. The methodology used in that document and, subsequently, in the analysis of hazards from the low-energy undulator test line (MOE 98) was also used for shielding analysis and dose determinations in this document. The hazards and potential consequences of storage ring (SR) operation covered in (MOE 94) still apply to non-top-up operations of the SR.

Two additional issues relevant to top-up operation, which give rise to potential radiological considerations, are 1) the possible use of the vertical scraper in the booster-to-storage ring (BTS) line to control the amount of charge that is being delivered to the storage ring, and 2) the potential accident situations, which give rise to radiation doses to individuals on the experiment hall floor and SR roof. By introducing the scraper, a portion of the beam produces a shower in the tungsten scraper, which leads to radiation fields on the top of the SR tunnel and in the Early Assembly Area (EAA). This requires additional shielding of the scraper. Potential doses to individuals on the floor of the experiment hall can result from a loss of particles down a photon beamline. Tracking results (COR 98) have shown that particles that find their way down a photon beamline will eventually be lost either in a $\mathrm{Pb}$ collimator or in the safety shutters in the front end of a bending magnet (BM), or on the safety shutters in an insertion device (ID) beamline. Either of these situations will result in a significant dose rate on the experiment hall floor if the event takes place during injection for top-up. Analyses of these and other accident scenarios are treated in this report. Minor doses will result from loss of a single stored beam in either of the above scenarios.

\section{Vertical Scraper Additional Shielding}

The vertical scraper is located in the BTS line, shortly after it enters the SR tunnel (Figure 1). The scraper is formed by two pieces of tungsten, which are moved into the beam from above and below. Each $\mathrm{W}$ block is $6.35 \mathrm{~cm}$ thick in the beam direction and $5.08 \mathrm{~cm}$ by $5.08 \mathrm{~cm}$ in the transverse directions. The block is thick enough to insure full shower development if the positron (electron) beam is totally intercepted. Radiation produced in the scraper is initially shielded in the vertical direction by $1 \mathrm{~m}$ of concrete in the tunnel roof. The dose point is taken as $3.343 \mathrm{~m}$ above the scraper. In the horizontal direction (toward the EAA), the shielding consists of $1.1 \mathrm{~m}$ of concrete, and the distance to the dose point is $6 \mathrm{~m}$ ( $\mathrm{A}$ in the Figure). 


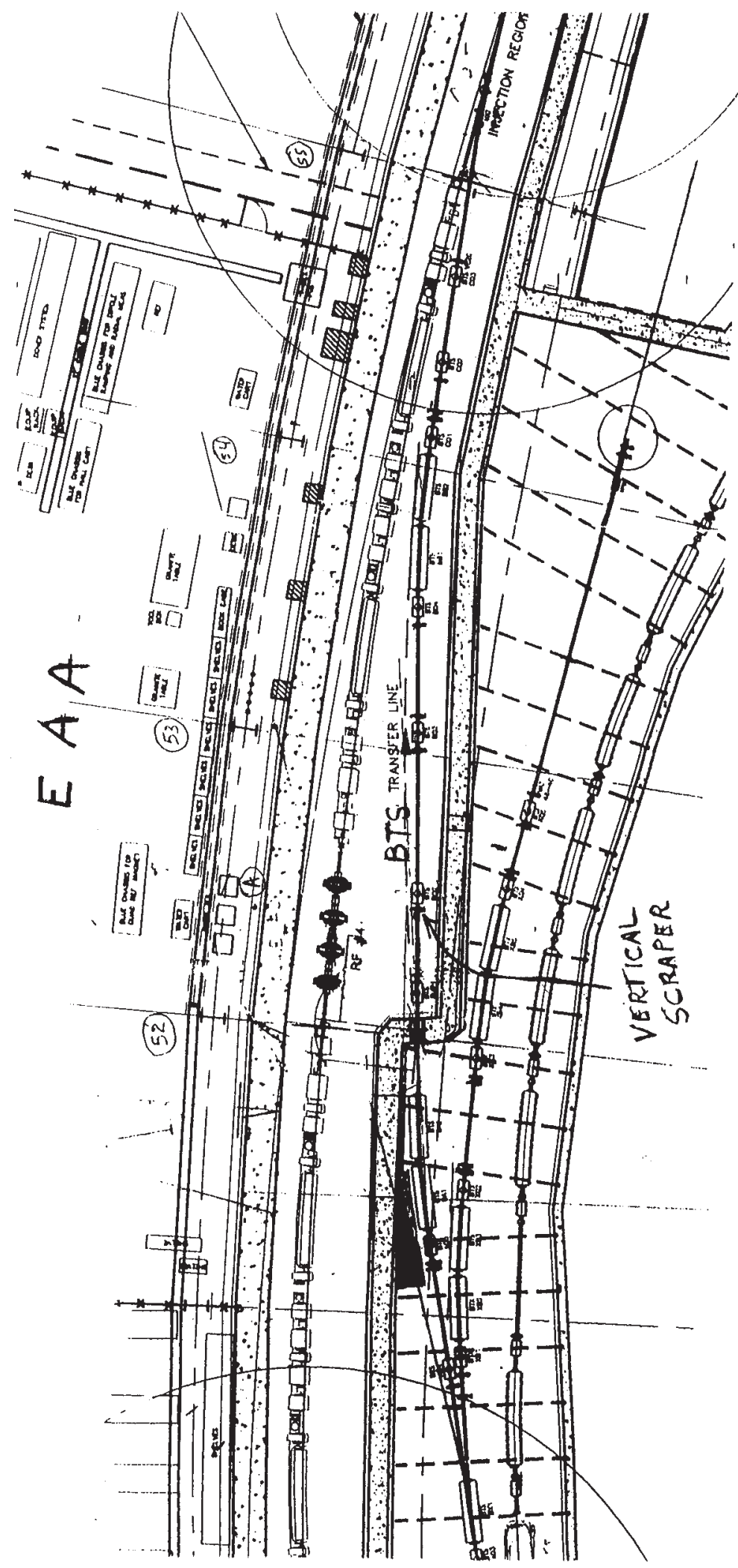

Figure 1. Location of Vertical Scraper in BTS Line 
To estimate the additional shielding needed for the scraper, tests were performed during two study periods in Run 1 of 1998. Radiation dose rates were measured on the SR roof and walkway and along the EAA wall at beam height. A reading of $5 \mathrm{mrem} / \mathrm{h} \gamma$ was obtained through the concrete roof of the SR in the vicinity above the scraper location, up to $0.7 \mathrm{mR} / \mathrm{h} \gamma$ on the walkway of the SR, and readings up to $1.85 \mathrm{mR} / \mathrm{h} \gamma$ through the EAA wall. The synchrotron was being run at $7 \mathrm{GeV}$ and 1$1.3 \mathrm{nC} /$ pulse at a $2-\mathrm{Hz}$ rate. For the second test, approximately $5.08 \mathrm{~cm}$ of Pb shielding was placed on the EAA side of the scraper. Dose rates for operation at 0.9-1.1 nC/pulse for $7 \mathrm{GeV}$ remained about the same for the readings on the SR roof, but the highest reading on the EAA wall decreased by a factor of about 6 . In addition, a reading of the neutron field at the surface of the SR roof, approximately directly above the scraper, was $12 \mathrm{mrem} / \mathrm{h}$. Measurements made with the scraper fully open indicated $50 \mu \mathrm{R} / \mathrm{h} \gamma$ on the roof above the scraper, $\leq 25 \mu \mathrm{R} / \mathrm{h} \gamma$ on the walkway, and $\leq 15 \mu \mathrm{R} / \mathrm{h}$ $\gamma$ through the EAA wall. No neutron (n) fields were detected at these latter locations during these measurements.

Based upon these results, the additional shielding around the scraper consists of $10.16 \mathrm{~cm}$ of $\mathrm{Pb}$ on the EAA side and on the top of the scraper, and $15.24 \mathrm{~cm}$ of polyethylene on top of the scraper. This will reduce the radiation fields to below desired levels when the scraper is in use during top-up.

\section{Top-Up Accidental Losses}

In Section 10 of (MOE 94), a number of potential loss incidents were evaluated with respect to their dose consequences for SR operation. In this section, incidents that may arise from top-up operations are treated. Some incidents have been evaluated during studies by measuring the doses produced when the beam is lost under certain scenarios. Other evaluations have been made by calculation only, assuming that the loss of beam occurs in a spill along the beamline. Based upon the empirical results obtained during missteering studies at the storage ring of the APS (DEC 97), a spill length of $4 \mathrm{~m}$ was used in the estimates of dose rates. This assumed spill length was shown to give conservative results by at least a factor of three in comparing calculated results with the measurements during missteerings. For some cases, the loss of beam can be considered to occur at a point. An example would be beam lost in a safety shutter, which is large enough to contain most of the shower that is produced.

\subsection{Stored Beam Losses}

\subsubsection{Loss on a Transition Piece of an ID Beamline}

Assuming that top-up is allowed only if a minimum amount of stored beam is present in the ring, studies were conducted to measure the dose consequences of losing the stored beam on a transition piece of an ID (TOP 97a, TOP 97b). In both studies, the storage ring was filled to approximately 100 $\mathrm{mA}$ of circulating current at $7 \mathrm{GeV}$ and then dumped on a transition piece, with the safety shutters both open and closed. Dose measurements were made both inside and outside of the first optics enclosure (FOE) for that particular ID. No $\gamma$ or $n$ radiation was measured inside or outside of the FOE when the loss occurred with the shutters closed. When the shutters were open, readings inside the FOE ranged from rems to tens of rems $(\gamma)$ and from mrems to tens of mrems (n) near the fixed 
mask, for each dump of the beam. The highest total dose during a dump, measured outside of the FOE, adjacent to the fixed mask, was $32 \mathrm{mrem}$ (neutron and gamma) for a loss of $100 \mathrm{~mA}$. For a stored beam at the safety envelope $(360 \mathrm{~mA}$ circulating current at $7 \mathrm{GeV})$, the total dose would be 115 mrem.

\subsubsection{Loss in the Front End of a Beamline}

A missteered particle beam will generally be unable to proceed farther than the collimator just past the second photon shutter in a BM beamline (Figure 2). However, it is possible that it could go as far as the safety shutters in a BM or an ID beamline (Figure 3). Assuming that the stored beam is missteered down a beamline, it may shower along the beamline or in the collimator for a BM line or in the safety shutters for a BM or an ID line. Because of the serpentine nature of the SR ratchet wall and the difference between the front-end components of a BM beamline and an ID beamline, the dose produced by a spill along the beamline in each case will be different. In some regions, the front-end beamline is only $50 \mathrm{~cm}$ from the $56-\mathrm{cm}$ thick ratchet wall, and in other cases it may be more than a meter away. For the spill analysis, 4-m-long segments were used to estimate the doses up to the collimator in the BM line. Similar 4-m segments in the ID line were used up the safety shutters. For the collimator and safety shutters, the doses were computed assuming point losses. Since the same type of safety shutters are used for both the BM and ID lines, and the geometry relative to the dose points is similar, the dose for the loss in the BM safety shutters should be comparable to that in the ID case. During top-up operation the safety shutters for a given beamline are open and no one is allowed in the FOE, so any bremsstrahlung radiation going down the photon beamline will be intercepted by the $\mathrm{Pb}$ beamstop at the back of the FOE. This case was considered in MOE 94 and was shown to result in a less than an MCI dose.

For the BM beamline, the doses computed at points A-D for a loss of a 360-mA stored beam along the front end are 97.6, 79.2, 31, and $53.4 \mathrm{mrem}$, respectively. The points correspond to the dose points for a 4-m spill at the beginning of the front end (A), where the distance from the wall is only about $50 \mathrm{~cm}$, to the dose point for a point loss in the collimator (D). For an ID beamline, the doses for points E-G are 107.3, 79.1, and $85.8 \mathrm{mrem}$, respectively. The points correspond to the dose points for a 4-m spill at the beginning of the front end $(\mathrm{E})$ to the dose point for a point loss in the safety shutter $(\mathrm{G})$. The dose between points $\mathrm{E}$ and $\mathrm{F}$ decreases because the distance from the beamline to the ratchet wall increases, resulting in the dose point being farther away. The dose for a point loss in the safety shutters for a BM line will be about the same as that for the ID line.

\subsection{Injected Beam Losses - At the Safety Envelope}

\subsubsection{Loss on a Transition Piece of an ID Beamline}

From the results of the measurements made during top-up studies (TOP 97a), for the case of beam being continuously lost on a transition piece during injection while the shutters are open, the dose per $\mathrm{nC}$ lost, outside of the FOE adjacent to the fixed collimator, turned out to be $0.087 \mathrm{mrem}$. At safety envelope operation $(7 \mathrm{GeV}, 20 \mathrm{nC} /$ pulse, $2 \mathrm{~Hz}$ ), this projects to $12.5 \mathrm{rem} / \mathrm{h}$, but at the proposed top-up operation maximum injection rate of $2 \mathrm{nC} / \mathrm{pulse}$, the dose rate is $1.25 \mathrm{rem} / \mathrm{h}$. 

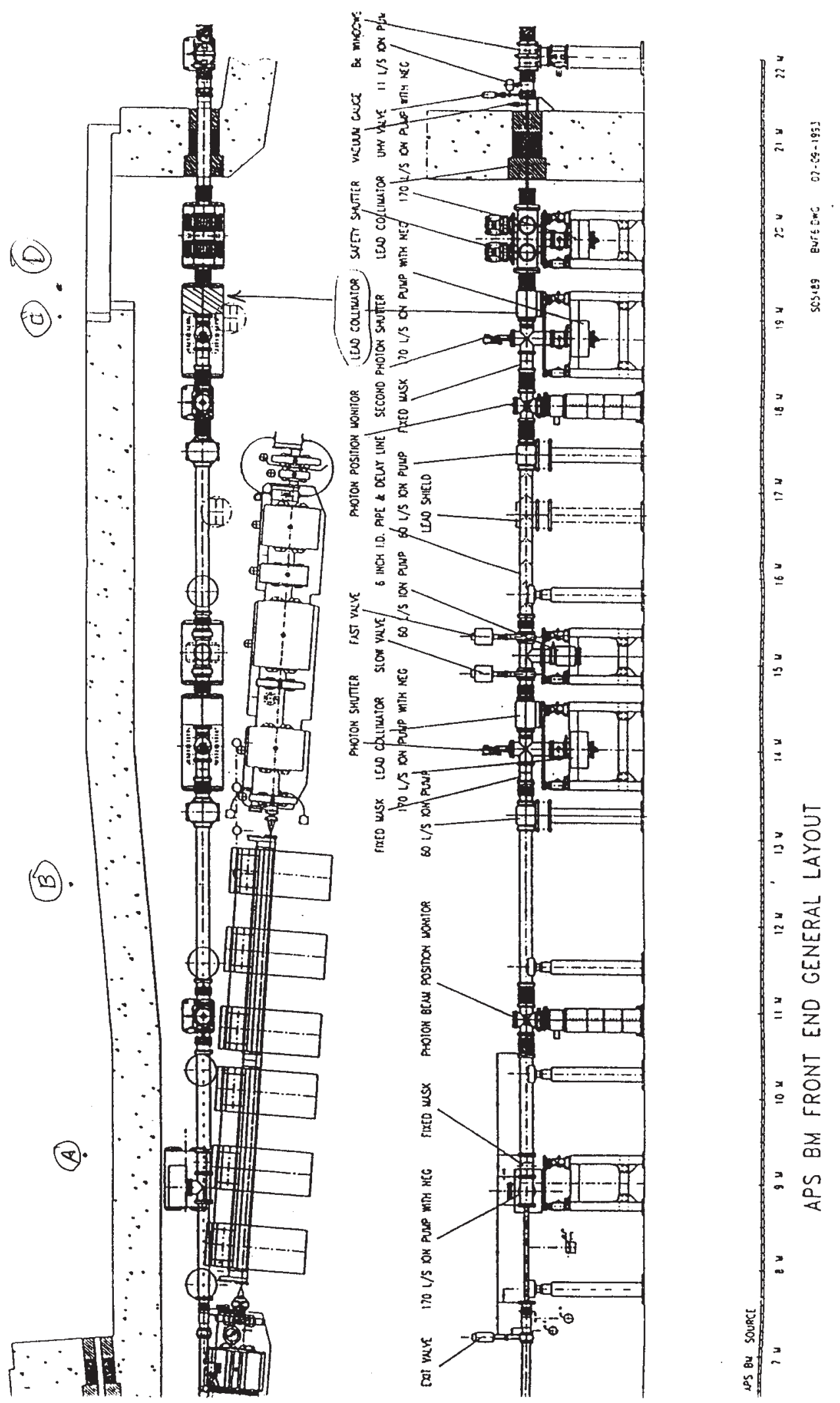

Figure 2. APS BM Front End General Layout 


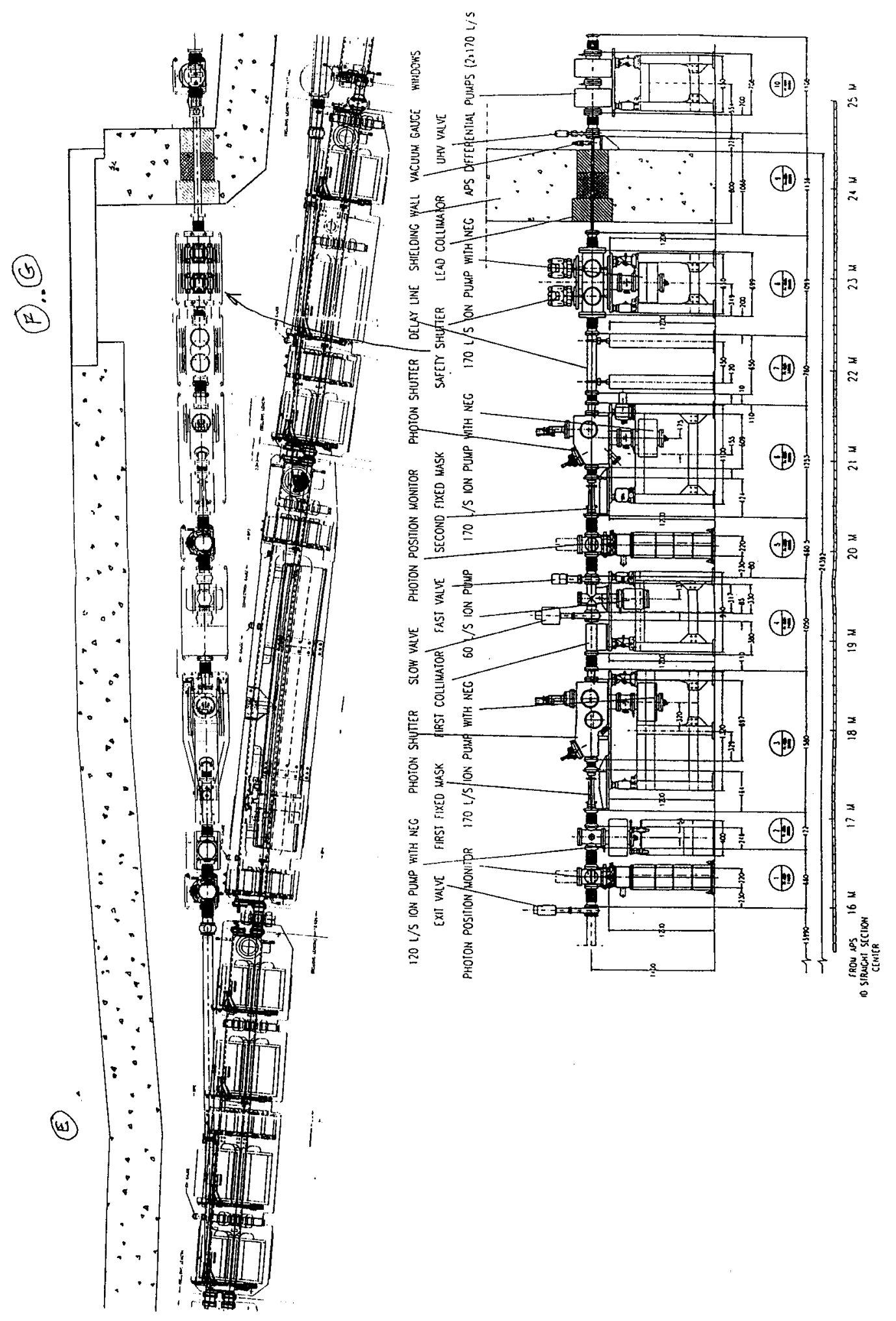

Figure 3. APS ID Front End General Layout 


\subsubsection{Loss in the Front End of a Beamline}

The same dose point locations in Figures 2 and 3 for the stored beam losses were also used for the losses during injection. For the BM line, the dose rates for points A-D are 10.6, 8.6, 3.37, and 5.81 $\mathrm{rem} / \mathrm{h}$, respectively. For the ID line dose points, the dose rates for points E-G are 11.7, 8.6, and 9.3 $\mathrm{rem} / \mathrm{h}$, respectively. Again, for a loss in the safety shutters of a BM line, the dose rate should be comparable to that for a loss in the ID safety shutters.

\subsection{Loss During Normal Top-Up Operation}

The performance goal for the APS is a beam lifetime of $10 \mathrm{~h}$. Assuming the total beam is lost during this time, the loss rate is 0.1 per $\mathrm{h}$. For a $360-\mathrm{mA}$ circulating current, the total charge in the ring is $1324.8 \mathrm{nC}$. The loss rate is then $132.48 \mathrm{nC} / \mathrm{h}$ or $0.0368 \mathrm{nC} / \mathrm{s}$. The initial goal of top-up is to control the charge between 360.36 and $360 \mathrm{~mA}$ ( 1 part in a thousand). For the SR, $1 \mathrm{~mA}$ circulating current represents $3.68 \mathrm{nC}$, so $0.36 \mathrm{~mA}$ equals $1.325 \mathrm{nC}$. Assuming the scraper is set so that for a pulse of $2 \mathrm{nC}$, the scraper allows only $1.325 \mathrm{nC}$ to pass through to the SR; then the circulating current rises to 360.36 and will decay back down to $360 \mathrm{~mA}$ in approximately $36 \mathrm{~s}$. For this operating scenario, a missteering will result in the loss of $0.2576 \mathrm{~W}$ in a 4-m-long spill. Scaling the results in Section 3.2.2, the dose rates for points A-D for the BM line are 8.9, 7.2, 2.8, and $4.9 \mathrm{mrem} / \mathrm{h}$, respectively. For the ID line, the dose rates for points E-G are $9.8,7.2$, and $7.8 \mathrm{mrem} / \mathrm{h}$, respectively. At the present operating condition of $100 \mathrm{~mA}$, the loss rate would be $1.022 \times 10^{-2} \mathrm{nC} / \mathrm{s}$, and the $0.1 \mathrm{~mA}$ would decay in $36 \mathrm{~s}$. This represents a power loss of $0.072 \mathrm{~W}$. For a spill under this condition, the dose rates for points A-D for the BM line are 2.5, 2.0, 0.8, and $1.4 \mathrm{mrem} / \mathrm{h}$, respectively. For the ID beamline, the dose rates for points E-G are $2.7,2.0$, and $2.2 \mathrm{mrem} / \mathrm{h}$, respectively.

The actual operating scenario may differ in practice from that stated above. The use of the scraper to control the charge going into the SR or the ability to inject smaller charges per pulse, as well as the reduced loss rate for the demonstrated beam lifetime of over $30 \mathrm{~h}$, should reduce the required average charge rate to even less than $1.022 \times 10^{-2} \mathrm{nC} / \mathrm{s}$. This would result in dose rates even less than those computed above, for a spill along a BM or ID beamline.

\section{REFERENCES}

COR 98 J. Corbett and A. Garren, "Report of APS Top-Up Tracking Review Committee," memo to J. Galayda from J. Corbett (SLAC) and A. Garren (UCLA), review team, Feb. 1998.

DEC 97 G. Decker et al., "'Maximal Credible Accident' Simulation Studies at the Storage Ring of the APS," Light Source Note LS-262, Argonne National Laboratory, Argonne, IL (1997).

MOE 94 H. J. Moe, unpublished information (July 31, 1994). Revised Sep. 24, 1997. 
MOE 98 H. J. Moe, "Radiological Considerations in the Operation of the Low Energy Undulator Test Line," Light Source Note LS-272, Argonne National Laboratory, Argonne, IL (1998).

TOP 97a H. J. Moe et al., unpublished information, March 17, 1997.

TOP 97b H. J. Moe et al., unpublished information, Sep. 18, 1997. 